\title{
Soft A-sets and Soft B-sets in Soft Topological Spaces
}

\author{
Naime Tozlu* and Şaziye Yüksel
}

(Communicated by İ. Onur KIYMAZ)

\begin{abstract}
In this paper, we introduce soft A-sets and soft B-sets in soft topological spaces which are defined over an initial universe with a fixed set of parameters and discuss their relationships with each other and other soft open sets like soft semiopen sets, soft preopen sets and soft $\alpha$-open sets with the help of counterexamples. We also introduce the concepts of soft A-continuous functions and soft B-continuous functions. Finally, we obtain decompositions of soft continuity: $f_{p u}$ is a soft continuous function if and only if it is both soft $\alpha$-continuous and soft A-continuous and also $f_{p u}$ is a soft continuous function if and only if it is both soft precontinuous and soft B-continuous.

Keywords: Soft set, Soft topological space, Soft A-set, Soft B-set, Soft A-continuous function, Soft B-continuous function. AMS Subject Classification (2010): 54A10; 54A20; 54C08.

${ }^{*}$ Corresponding author
\end{abstract}

\section{Introduction}

The concept of soft sets was initiated by Molodtsov [10] in 1999 as a completely new approach for modeling vagueness and uncertainty. He has shown several applications of this theory in solving many practical problems in economics, engineering, social science, medical science, etc. Later Maji et al. [8] presented some new definitions on soft sets such as a subset, the complement of a soft set. Research works on soft sets are progressing rapidly in recent years.

Shabir and Naz [11] introduced the soft topological spaces which are defined over an initial universe with a fixed set of parameters. Later Aygünoğlu and Aygün [3], Min [9], Zorlutuna et al. [13] and Hussain and Ahmad [5] continued to study the properties of soft topological spaces. They got many important results in soft topological spaces.

Recently, weak forms of soft open sets were studied. First, Chen [4] investigated soft semiopen sets in soft topological spaces and studied some properties of them. Later Arockiarani and Arokialancy [2] defined soft $\beta$ open sets and some other weak forms of soft open sets in soft topological spaces. Finally, soft $\alpha$-open sets and soft $\alpha$-continuous functions in soft topological spaces were introduced by Akdağ and Özkan [1].

In the present study, we introduce some new concepts in soft topological spaces which are defined over an initial universe with a fixed set of parameters such as soft A-sets and soft B-sets which are weak forms of soft open sets. We study the relationships between different types of soft open sets in soft topological spaces. We also introduce the concepts of soft A-continuous and soft B-continuous functions. Finally, we give some decompositions of soft continuity.

\section{Preliminaries}

In this section, we present the basic definitions and results of soft set theory which may be found in earlier studies.

Received : 28-01-2017, Accepted : 11-04-2017 
Let $X$ be an initial universe set and $E$ be the set of all possible parameters with respect to $X$. Parameters are often attributes, characteristics or properties of the objects in $X$. Let $P(X)$ denote the power set of $X$. Then a soft set over $X$ is defined as follows.

Definition 2.1. [10] A pair $(F, A)$ is called a soft set over $X$ where $A \subseteq E$ and $F: A \rightarrow P(X)$ is a set valued mapping. In other words, a soft set over $X$ is a parameterized family of subsets of the universe $X$. For $\forall \varepsilon \in A$, $F(\varepsilon)$ may be considered as the set of $\varepsilon$-approximate elements of the soft set $(F, A)$. It is worth noting that $F(\varepsilon)$ may be arbitrary. Some of them may be empty, and some may have nonempty intersection.

The set of all soft sets over $X$ is denoted by $S S(X)_{E}$.

Definition 2.2. [8] A soft set $(F, A)$ over $X$ is said to be a null soft set denoted by $\Phi$ if for all $e \in A, F(e)=\emptyset$. A soft set $(F, A)$ over $X$ is said to be an absolute soft set denoted by $\tilde{A}$ if for all $e \in A, F(e)=X$.

Definition 2.3. [11] Let $Y$ be a nonempty subset of $X$, then $\tilde{Y}$ denotes the soft set $(Y, E)$ over $X$ for which $Y(e)=Y$, for all $e \in E$. In particular, $(X, E)$ will be denoted by $\tilde{X}$.

Definition 2.4. [8] For two soft sets $(F, A)$ and $(G, B)$ over $X$, we say that $(F, A)$ is a soft subset of $(G, B)$ if $A \subseteq B$ and for all $e \in A, F(e)$ and $G(e)$ are identical approximations. We write $(F, A) \sqsubseteq(G, B)$. $(F, A)$ is said to be a soft super set of $(G, B)$, if $(G, B)$ is a soft subset of $(F, A)$. We denote it by $(G, B) \sqsubseteq(F, A)$. Then $(F, A)$ and $(G, B)$ are said to be soft equal if $(F, A)$ is a soft subset of $(G, B)$ and $(G, B)$ is a soft subset of $(F, A)$.

Definition 2.5. [8] The union of two soft sets $(F, A)$ and $(G, B)$ over $X$ is the soft set $(H, C)$, where $C=A \cup B$ and for all $e \in C, H(e)=F(e)$ if $e \in A \backslash B, H(e)=G(e)$ if $e \in B \backslash A, H(e)=F(e) \cup G(e)$ if $e \in A \cap B$. We write $(F, A) \sqcup(G, B)=(H, C)$.

Definition 2.6. [8] The intersection $(H, C)$ of two soft sets $(F, A)$ and $(G, B)$ over $X$, denoted by $(F, A) \sqcap(G, B)$, is defined as $C=A \cap B$, and $H(e)=F(e) \cap G(e)$ for all $e \in C$.

Definition 2.7. [11] The difference $(H, E)$ of two soft sets $(F, E)$ and $(G, E)$ over $X$, denoted by $(F, E) \backslash(G, E)$, is defined as $H(e)=F(e) \backslash G(e)$ for all $e \in E$.

Definition 2.8. [11] The relative complement of a soft set $(F, E)$ is denoted by $(F, E)^{c}$ and is defined by $(F, E)^{c}=$ $\left(F^{c}, E\right)$ where $F^{c}: E \longrightarrow P(X)$ is a mapping given by $F^{c}(e)=X \backslash F(e)$ for all $e \in E$.

Definition 2.9. [13] The soft set $(F, E) \in S S(X)_{E}$ is called a soft point in $\tilde{X}$, denoted by $x_{e}$, if for the element $e \in E, F(e) \neq \emptyset$ and $F\left(e^{\prime}\right)=\emptyset$ for all $e^{\prime} \in E \backslash\{e\}$. The soft point $x_{e}$ is said to be in the soft set $(G, E)$, denoted by $x_{e} \in(G, E)$, if for the element $e \in E$ and $F(e) \subseteq G(e)$.

Definition 2.10. [11] Let $\tau$ be the collection of soft sets over $X$, then $\tau$ is said to be a soft topology on $X$ if

(1) $\Phi, \tilde{X} \in \tau$

(2) If $(F, E),(G, E) \in \tau$, then $(F, E) \sqcap(G, E) \in \tau$

(3) If $\left\{\left(F_{i}, E\right)\right\}_{i \in I} \in \tau, \forall i \in I$, then $\sqcup_{i \in I}\left(F_{i}, E\right) \in \tau$.

The triplet $(X, \tau, E)$ is called a soft topological space over $X$. Every member of $\tau$ is called a soft open set in $X$. A soft set $(F, E)$ over $X$ is called soft closed set in $X$ if its relative complement $(F, E)^{c}$ belongs to $\tau$. We will denote the family of all soft open sets (resp., soft closed sets) of a soft topological space $(X, \tau, E)$ by $\operatorname{SOS}(X, \tau, E)$ (resp., $\operatorname{SCS}(X, \tau, E))$.

Throughout the paper, the spaces $X$ and $Y$ (or $(X, \tau, E)$ and $(Y, \nu, K))$ stand for soft topological spaces assumed unless stated otherwise.

Definition 2.11. Let $(X, \tau, E)$ be a soft topological space over $X$ and $(F, E)$ be a soft set over $X$.

(1) [11] The soft closure of $(F, E)$ is the soft set $c l(F, E)=\sqcap\{(G, E):(G, E)$ is soft closed and $(F, E) \sqsubseteq(G, E)\}$.

(2) [13] The soft interior of $(F, E)$ is the soft set $\operatorname{int}(F, E)=\sqcup\{(H, E):(H, E)$ is soft open and $(H, E) \sqsubseteq(F, E)\}$.

Clearly, $\operatorname{cl}(F, E)$ is the smallest soft closed set over $X$ which contains $(F, E)$ and $\operatorname{int}(F, E)$ is the largest soft open set over $X$ which is contained in $(F, E)$. 
Theorem 2.1. [11] Let $(X, \tau, E)$ be a soft topological space and $(F, E),(G, E)$ soft sets over $X$. Then

(1) $\operatorname{cl}(\Phi)=\Phi$ and $\operatorname{cl}(\tilde{X})=\tilde{X}$.

(2) $(F, E) \sqsubseteq c l(F, E)$.

(3) $(F, E)$ is a soft closed set if and only if $(F, E)=\operatorname{cl}(F, E)$.

(4) $\operatorname{cl}(\operatorname{cl}(F, E))=\operatorname{cl}(F, E)$.

(5) $(F, E) \sqsubseteq(G, E)$ implies $c l(F, E) \sqsubseteq \operatorname{cl}(G, E)$.

(6) $\operatorname{cl}((F, E) \sqcup(G, E))=\operatorname{cl}(F, E) \sqcup \operatorname{cl}(G, E)$.

(7) $\operatorname{cl}((F, E) \sqcap(G, E)) \sqsubseteq c l(F, E) \sqcap \operatorname{cl}(G, E)$.

Theorem 2.2. [5] Let $(X, \tau, E)$ be a soft topological space and $(F, E),(G, E)$ soft sets over $X$. Then

(1) $\operatorname{int}(\Phi)=\Phi$ and $\operatorname{int}(\tilde{X})=\tilde{X}$.

(2) $\operatorname{int}(F, E) \sqsubseteq(F, E)$.

(3) $(F, E)$ is a soft open set if and only if $(F, E)=\operatorname{int}(F, E)$.

(4) $\operatorname{int}(\operatorname{int}(F, E))=\operatorname{int}(F, E)$.

$(5)(F, E) \sqsubseteq(G, E)$ implies $\operatorname{int}(F, E) \sqsubseteq \operatorname{int}(G, E)$.

(6) $\operatorname{int}((F, E) \sqcap(G, E))=\operatorname{int}(F, E) \sqcap \operatorname{int}(G, E)$.

$(7) \operatorname{int}((F, E) \sqcup(G, E)) \sqsupseteq \operatorname{int}(F, E) \sqcup \operatorname{int}(G, E)$.

Definition 2.12. [4] A soft set $(F, E)$ is called soft semiopen set in a soft topological space $X$ if $(F, E) \sqsubseteq \operatorname{cl}(\operatorname{int}(F, E))$. The relative complement of a soft semiopen set is called a soft semiclosed set.

Definition 2.13. [2] A soft set $(F, E)$ is called soft preopen set in a soft topological space $X$ if $(F, E) \sqsubseteq \operatorname{int}(c l(F, E))$. The relative complement of a soft preopen set is called a soft preclosed set.

Definition 2.14. [1] A soft set $(F, E)$ is called soft $\alpha$-open set in a soft topological space $X$ if $(F, E) \sqsubseteq \operatorname{int}(\operatorname{cl}(\operatorname{int}(F, E)))$. The relative complement of a soft $\alpha$-open set is called a soft $\alpha$-closed set.

We will denote the family of all soft $\alpha$-open sets (resp., soft preopen sets and soft semiopen sets) of a soft topological space $(X, \tau, E)$ by $\operatorname{S} \alpha \mathrm{OS}(X, \tau, E)$ (resp., $\operatorname{SPOS}(X, \tau, E)$ and $\operatorname{SSOS}(X, \tau, E))$.

Remark 2.1. [1] It is obvious that every soft open (resp., soft closed) set is a soft $\alpha$-open (soft $\alpha$-closed) set. Similarly, every soft $\alpha$-open set is soft preopen and soft semiopen.

The example given below show that the converses of these implications are not true.

Example 2.1. [1] Let $X=\left\{x_{1}, x_{2}, x_{3}, x_{4}\right\}, E=\left\{e_{1}, e_{2}, e_{3}\right\}$ and $\tau=\left\{\Phi, \tilde{X},\left(F_{1}, E\right),\left(F_{2}, E\right), \ldots,\left(F_{15}, E\right)\right\}$ where $\left(F_{1}, E\right),\left(F_{2}, E\right), \ldots,\left(F_{15}, E\right)$ are soft sets over $X$, defined as follows:

$$
\begin{aligned}
\left(F_{1}, E\right) & =\left\{\left(e_{1},\left\{x_{1}\right\}\right),\left(e_{2},\left\{x_{2}, x_{3}\right\}\right),\left(e_{3},\left\{x_{1}, x_{4}\right\}\right)\right\} \\
\left(F_{2}, E\right) & =\left\{\left(e_{1},\left\{x_{2}, x_{4}\right\}\right),\left(e_{2},\left\{x_{1}, x_{3}, x_{4}\right\}\right),\left(e_{3},\left\{x_{1}, x_{2}, x_{4}\right\}\right)\right\} \\
\left(F_{3}, E\right) & =\left\{\left(e_{1}, \emptyset\right),\left(e_{2},\left\{x_{3}\right\}\right),\left(e_{3},\left\{x_{1}\right\}\right)\right\} \\
\left(F_{4}, E\right) & =\left\{\left(e_{1},\left\{x_{1}, x_{2}, x_{4}\right\}\right),\left(e_{2}, X\right),\left(e_{3}, X\right)\right\} \\
\left(F_{5}, E\right) & =\left\{\left(e_{1},\left\{x_{1}, x_{3}\right\}\right),\left(e_{2},\left\{x_{2}, x_{4}\right\}\right),\left(e_{3},\left\{x_{2}\right\}\right)\right\} \\
\left(F_{6}, E\right) & =\left\{\left(e_{1},\left\{x_{1}\right\}\right),\left(e_{2},\left\{x_{2}\right\}\right),\left(e_{3}, \emptyset\right)\right\} \\
\left(F_{7}, E\right) & =\left\{\left(e_{1},\left\{x_{1}, x_{3}\right\}\right),\left(e_{2},\left\{x_{2}, x_{3}, x_{4}\right\}\right),\left(e_{3},\left\{x_{1}, x_{2}, x_{4}\right\}\right)\right\} \\
\left(F_{8}, E\right) & =\left\{\left(e_{1}, \emptyset\right),\left(e_{2},\left\{x_{4}\right\}\right),\left(e_{3},\left\{x_{2}\right\}\right)\right\} \\
\left(F_{9}, E\right) & =\left\{\left(e_{1}, X\right),\left(e_{2}, X\right),\left(e_{3},\left\{x_{1}, x_{2}, x_{3}\right\}\right)\right\} \\
\left(F_{10}, E\right) & =\left\{\left(e_{1},\left\{x_{1}, x_{3}\right\}\right),\left(e_{2},\left\{x_{2}, x_{3}, x_{4}\right\}\right),\left(e_{3},\left\{x_{1}, x_{2}\right\}\right)\right\} \\
\left(F_{11}, E\right) & =\left\{\left(e_{1},\left\{x_{2}, x_{3}, x_{4}\right\}\right),\left(e_{2}, X\right),\left(e_{3},\left\{x_{1}, x_{2}, x_{3}\right\}\right)\right\} \\
\left(F_{12}, E\right) & =\left\{\left(e_{1},\left\{x_{1}\right\}\right),\left(e_{2},\left\{x_{2}, x_{3}, x_{4}\right\}\right),\left(e_{3},\left\{x_{1}, x_{2}, x_{4}\right\}\right)\right\} \\
\left(F_{13}, E\right) & =\left\{\left(e_{1},\left\{x_{1}\right\}\right),\left(e_{2},\left\{x_{2}, x_{4}\right\}\right),\left(e_{3},\left\{x_{2}\right\}\right)\right\} \\
\left(F_{14}, E\right) & =\left\{\left(e_{1},\left\{x_{3}, x_{4}\right\}\right),\left(e_{2},\left\{x_{1}, x_{2}\right\}\right),\left(e_{3}, \emptyset\right)\right\} \\
\left(F_{15}, E\right) & =\left\{\left(e_{1},\left\{x_{1}\right\}\right),\left(e_{2},\left\{x_{2}, x_{3}\right\}\right),\left(e_{3},\left\{x_{1}\right\}\right)\right\}
\end{aligned}
$$


Then $\tau$ defines a soft topology on $X$ and thus $(X, \tau, E)$ is a soft topological space over $X$. Then $\left(G_{1}, E\right)=$ $\left\{\left(e_{1},\left\{x_{1}, x_{2}, x_{3}\right\}\right),\left(e_{2},\left\{x_{2}, x_{3}, x_{4}\right\}\right),\left(e_{3},\left\{x_{1}, x_{2}\right\}\right)\right\}$ is a soft $\alpha$-open set in $X$ but not soft open. $\left(G_{2}, E\right)=\left\{\left(e_{1},\left\{x_{2}\right\}\right)\right.$, $\left.\left(e_{2},\left\{x_{3}\right\}\right),\left(e_{3},\left\{x_{1}, x_{3}\right\}\right)\right\}$ is a soft semiopen set in $X$ but not soft $\alpha$-open. $\left(G_{3}, E\right)=\left\{\left(e_{1}, \emptyset\right),\left(e_{2}, \emptyset\right),\left(e_{3},\left\{x_{2}\right\}\right)\right\}$ is a soft preopen set in $X$ but not soft $\alpha$-open.

Definition 2.15. [12] Let $(X, \tau, E)$ be a soft topological space. A soft set $(F, E)$ is called soft regular open set in $X$ if $(F, E)=\operatorname{int}(\operatorname{cl}(F, E))$. The relative complement of a soft regular open set is called a soft regular closed set.

We will denote the family of all soft regular open sets (resp., soft regular closed sets) of a soft topological space $(X, \tau, E)$ by $\operatorname{SROS}(X, \tau, E)$ (resp., $\operatorname{SRCS}(X, \tau, E))$.

Remark 2.2. [12] Every soft regular open set in a soft topological space $(X, \tau, E)$ is soft open.

Now, we give an example to show that the converse of Remark 2.2 does not hold.

Example 2.2. [12] Let $X=\left\{x_{1}, x_{2}, x_{3}, x_{4}\right\}, E=\left\{e_{1}, e_{2}\right\}$ and $\tau=\left\{\Phi, \tilde{X},\left(F_{1}, E\right),\left(F_{2}, E\right),\left(F_{3}, E\right)\right\}$ where $\left(F_{1}, E\right)$, $\left(F_{2}, E\right),\left(F_{3}, E\right)$ are soft sets over $X$, defined as follows:

$$
\begin{aligned}
& \left(F_{1}, E\right)=\left\{\left(e_{1},\left\{x_{1}\right\}\right),\left(e_{2},\left\{x_{2}\right\}\right)\right\}, \\
& \left(F_{2}, E\right)=\left\{\left(e_{1},\left\{x_{1}, x_{2}\right\}\right),\left(e_{2},\left\{x_{1}, x_{2}, x_{3}\right\}\right)\right\}, \\
& \left(F_{3}, E\right)=\left\{\left(e_{1},\left\{x_{1}, x_{2}, x_{4}\right\}\right),\left(e_{2}, X\right)\right\} .
\end{aligned}
$$

Then $\tau$ defines a soft topology on $X$ and thus $(X, \tau, E)$ is a soft topological space over $X$. Then $\left(F_{2}, E\right)$ is a soft open set in $X$ but not soft regular open.

Definition 2.16. [6] Let $S S(X)_{E}$ and $S S(Y)_{K}$ be families of soft sets, $u: X \longrightarrow Y$ and $p: E \longrightarrow K$ be mappings. Then the mapping $f_{p u}: S S(X)_{E} \longrightarrow S S(Y)_{K}$ is defined as:

(1) Let $(F, E) \in S S(X)_{E}$. The image of $(F, E)$ under $f_{p u}$, written as $f_{p u}(F, E)=\left(f_{p u}(F), p(E)\right)$, is a soft set in $S S(Y)_{K}$ such that

$$
f_{p u}(F)(y)=\left\{\begin{array}{lr}
\cup_{x \in p^{-1}(y) \cap A} u(F(x)) & , p^{-1}(y) \cap A \neq \emptyset \\
\emptyset & \text {, otherwise }
\end{array}\right.
$$

for all $y \in K$.

(2) Let $(G, K) \in S S(Y)_{K}$. The inverse image of $(G, K)$ under $f_{p u}$, written as $f_{p u}^{-1}(G, K)=\left(f_{p u}^{-1}(G), p^{-1}(K)\right)$, is a soft set in $S S(X)_{E}$ such that

for all $x \in E$.

$$
f_{p u}^{-1}(G)(x)= \begin{cases}u^{-1}(G(p(x))) & , p(x) \in K \\ \emptyset & , \text { otherwise }\end{cases}
$$

Definition 2.17. [13] Let $(X, \tau, E)$ and $(Y, v, K)$ be soft topological spaces and $f_{p u}: S S(X)_{E} \longrightarrow S S(Y)_{K}$ be a function. Then $f_{p u}$ is called a soft continuous function if for each $(G, K) \in S O S(Y)$ we have $f_{p u}^{-1}(G, K) \in S O S(X)$.

Definition 2.18. [7] Let $(X, \tau, E)$ and $(Y, v, K)$ be soft topological spaces and $f_{p u}: S S(X)_{E} \longrightarrow S S(Y)_{K}$ be a function. Then $f_{p u}$ is called a soft semicontinuous function if for each $(G, K) \in S O S(Y)$ we have $f_{p u}^{-1}(G, K) \in$ $\operatorname{SSOS}(X)$.

Definition 2.19. [1] Let $(X, \tau, E)$ and $(Y, v, K)$ be soft topological spaces and $f_{p u}: S S(X)_{E} \longrightarrow S S(Y)_{K}$ be a function. Then $f_{p u}$ is called a soft $\alpha$-continuous function if for each $(G, K) \in S O S(Y)$ we have $f_{p u}^{-1}(G, K) \in$ $S \alpha O S(X)$.

Definition 2.20. [1] Let $(X, \tau, E)$ and $(Y, v, K)$ be soft topological spaces and $f_{p u}: S S(X)_{E} \longrightarrow S S(Y)_{K}$ be a function. Then $f_{p u}$ is called a soft precontinuous function if for each $(G, K) \in S O S(Y)$ we have $f_{p u}^{-1}(G, K) \in$ $\operatorname{SPOS}(X)$.

Remark 2.3. [1] It is clear that every soft $\alpha$-continuous function is soft semicontinuous and soft precontinuous. Every soft continuous function is soft $\alpha$-continuous.

Theorem 2.3. [1] Let $(X, \tau, E)$ and $(Y, v, K)$ be soft topological spaces and $f_{p u}: S S(X)_{E} \longrightarrow S S(Y)_{K}$ be a function. If $f_{p u}$ is soft semicontinuous and soft precontinuous, then $f_{p u}$ is soft $\alpha$-continuous. 


\section{Soft A-sets and Soft B-sets}

Definition 3.1. Let $(X, \tau, E)$ be a soft topological space. A soft set $(F, E)$ is called soft A-set in $X$ if $(F, E)=$ $(G, E) \backslash(H, E)$, where $(G, E)$ is a soft open set and $(H, E)$ is a soft regular open set in $X$.

It is obvious that a soft set $(F, E)$ is a soft A-set if and only if $(F, E)=(G, E) \sqcap(K, E)$, where $(G, E)$ is a soft open set and $(K, E)$ is a soft regular closed set.

Theorem 3.1. In a soft topological space $(X, \tau, E)$, every soft open set is a soft A-set.

Proof. Let $(F, E)$ be a soft open set in $X$. Then $(F, E)$ is a soft A-set since $(F, E)=(F, E) \backslash \Phi$.

The converse of Theorem 3.1 is not true in general as shown in the following example.

Example 3.1. Let $X=\left\{x_{1}, x_{2}, x_{3}, x_{4}\right\}, E=\left\{e_{1}, e_{2}\right\}$ and $\tau=\left\{\Phi, \tilde{X},\left(F_{1}, E\right),\left(F_{2}, E\right), \ldots,\left(F_{11}, E\right)\right\}$ where $\left(F_{1}, E\right)$, $\left(F_{2}, E\right), \ldots,\left(F_{11}, E\right)$ are soft sets over $X$, defined as follows:

$$
\begin{aligned}
\left(F_{1}, E\right) & =\left\{\left(e_{1},\left\{x_{1}\right\}\right),\left(e_{2},\left\{x_{1}\right\}\right)\right\}, \\
\left(F_{2}, E\right) & =\left\{\left(e_{1},\left\{x_{2}\right\}\right),\left(e_{2},\left\{x_{2}\right\}\right)\right\}, \\
\left(F_{3}, E\right) & =\left\{\left(e_{1},\left\{x_{1}, x_{2}\right\}\right),\left(e_{2},\left\{x_{1}, x_{2}\right\}\right)\right\}, \\
\left(F_{4}, E\right) & =\left\{\left(e_{1},\left\{x_{1}, x_{2}, x_{3}\right\}\right),\left(e_{2},\left\{x_{1}, x_{3}\right\}\right)\right\}, \\
\left(F_{5}, E\right) & =\left\{\left(e_{1},\left\{x_{1}, x_{2}, x_{4}\right\}\right),\left(e_{2},\left\{x_{1}, x_{2}, x_{3}\right\}\right)\right\}, \\
\left(F_{6}, E\right) & =\left\{\left(e_{1},\left\{x_{2}\right\}\right),\left(e_{2}, \emptyset\right)\right\}, \\
\left(F_{7}, E\right) & =\left\{\left(e_{1},\left\{x_{1}, x_{2}\right\}\right),\left(e_{2},\left\{x_{1}\right\}\right)\right\}, \\
\left(F_{8}, E\right) & =\left\{\left(e_{1},\left\{x_{1}, x_{2}, x_{3}\right\}\right),\left(e_{2},\left\{x_{1}, x_{2}, x_{3}\right\}\right)\right\}, \\
\left(F_{9}, E\right) & =\left\{\left(e_{1}, X\right),\left(e_{2},\left\{x_{1}, x_{2}, x_{3}\right\}\right)\right\}, \\
\left(F_{10}, E\right) & =\left\{\left(e_{1},\left\{x_{1}, x_{2}\right\}\right),\left(e_{2},\left\{x_{1}, x_{2}, x_{3}\right\}\right)\right\}, \\
\left(F_{11}, E\right) & =\left\{\left(e_{1},\left\{x_{1}, x_{2}\right\}\right),\left(e_{2},\left\{x_{1}, x_{3}\right\}\right)\right\} .
\end{aligned}
$$

Then $\tau$ defines a soft topology on $X$ and thus $(X, \tau, E)$ is a soft topological space over $X$ [12].

Let $(G, E)$ be a soft set over $X$ such that $(G, E)=\left\{\left(e_{1},\left\{x_{2}, x_{3}\right\}\right),\left(e_{2},\left\{x_{3}\right\}\right)\right\}$. Since $(G, E)=\left(F_{4}, E\right) \backslash\left(F_{1}, E\right)$ such that $\left(F_{4}, E\right)$ is soft open and $\left(F_{1}, E\right)$ is soft regular open, $(G, E)$ is a soft A-set but not soft open.

Theorem 3.2. In a soft topological space $(X, \tau, E)$, every soft $A$-set is soft semiopen.

Proof. Let $(F, E)=(G, E) \sqcap(K, E)$ be a soft A-set, where $(G, E)$ is soft open and $(K, E)=\operatorname{cl}(\operatorname{int}(K, E))$. Since $(F, E)=(G, E) \sqcap(K, E)$, we have $\operatorname{int}(F, E) \sqsupseteq(G, E) \sqcap \operatorname{int}(K, E)$. It is easily seen that $\operatorname{int}(F, E) \sqsubseteq(F, E) \sqsubseteq(K, E)$, hence $\operatorname{int}(F, E)=\operatorname{int}(\operatorname{int}(F, E)) \sqsubseteq \operatorname{int}(K, E)$. But $\operatorname{int}(F, E) \sqsubseteq(F, E) \sqsubseteq(G, E)$, hence $\operatorname{int}(F, E) \sqsubseteq(G, E) \sqcap$ $\operatorname{int}(K, E)$. Therefore $\operatorname{int}(F, E)=(G, E) \sqcap \operatorname{int}(K, E)$. Now we prove $(F, E) \sqsubseteq \operatorname{cl}(\operatorname{int}(F, E))$. Let $x_{e} \in(F, E)$ and $(H, E)$ be an arbitrary soft open set containing $x_{e}$. Then $(G, E) \sqcap(H, E)$ is also a soft open set containing $x_{e}$. Since $x_{e} \in(K, E)=\operatorname{cl}(\operatorname{int}(K, E))$, there is a soft point $y_{e} \in \operatorname{int}(K, E)$ such that $x_{e} \neq y_{e}$ and $y_{e} \in(G, E) \sqcap(H, E)$. Hence $y_{e} \in(G, E) \sqcap \operatorname{int}(K, E)=\operatorname{int}(F, E)$. Therefore $x_{e} \in \operatorname{cl}(\operatorname{int}(F, E))$ and $(F, E) \sqsubseteq \operatorname{cl}(\operatorname{int}(F, E))$. Thus we obtain $(F, E)$ is soft semiopen in $X$.

The converse of Theorem 3.2 is not true in general as shown in the following example.

Example 3.2. Let $X=\left\{x_{1}, x_{2}, x_{3}\right\}, E=\left\{e_{1}, e_{2}\right\}$ and $\tau=\left\{\Phi, \tilde{X},\left(F_{1}, E\right),\left(F_{2}, E\right),\left(F_{3}, E\right)\right\}$ where $\left(F_{1}, E\right),\left(F_{2}, E\right)$, $\left(F_{3}, E\right)$ are soft sets over $X$, defined as follows:

$$
\begin{aligned}
& \left(F_{1}, E\right)=\left\{\left(e_{1},\left\{x_{1}\right\}\right),\left(e_{2},\left\{x_{1}\right\}\right)\right\}, \\
& \left(F_{2}, E\right)=\left\{\left(e_{1},\left\{x_{2}\right\}\right),\left(e_{2},\left\{x_{2}\right\}\right)\right\}, \\
& \left(F_{3}, E\right)=\left\{\left(e_{1},\left\{x_{1}, x_{2}\right\}\right),\left(e_{2},\left\{x_{1}, x_{2}\right\}\right)\right\} .
\end{aligned}
$$

Then $\tau$ defines a soft topology on $X$ and thus $(X, \tau, E)$ is a soft topological space over $X$.

Let $(G, E)$ be a soft set over $X$ such that $(G, E)=\left\{\left(e_{1},\left\{x_{1}\right\}\right),\left(e_{2},\left\{x_{1}, x_{3}\right\}\right)\right\}$. Since $(G, E) \sqsubseteq \operatorname{cl}(\operatorname{int}(G, E))=$ $\left(F_{2}, E\right)^{c},(G, E)$ is a soft semiopen set but not a soft A-set.

The examples below show that a soft $\alpha$-open set need not be a soft A-set and a soft A-set need not be a soft $\alpha$-open set. 
Example 3.3. Let $X=\left\{x_{1}, x_{2}, x_{3}, x_{4}\right\}$ and $E=\left\{e_{1}, e_{2}\right\}$. Let us take the soft topology $\tau$ on $X$ and the soft set $(G, E)$ in Example 3.1. Then, $(G, E)$ is a soft A-set but not soft $\alpha$-open.

Example 3.4. Let $X=\left\{x_{1}, x_{2}, x_{3}\right\}, E=\left\{e_{1}, e_{2}\right\}$ and $\tau=\{\Phi, \widetilde{X},(F, E)\}$ where $(F, E)$ is a soft set over $X$, defined as follows:

$$
(F, E)=\left\{\left(e_{1},\left\{x_{1}\right\}\right),\left(e_{2},\left\{x_{2}\right\}\right)\right\}
$$

Then $\tau$ defines a soft topology on $X$ and thus $(X, \tau, E)$ is a soft topological space over $X$.

Let $(G, E)$ be a soft set over $X$ such that $(G, E)=\left\{\left(e_{1},\left\{x_{1}, x_{2}\right\}\right),\left(e_{2},\left\{x_{2}\right\}\right)\right\}$. Since $(G, E) \sqsubseteq \operatorname{int}(\operatorname{cl}(\operatorname{int}(G, E)))=$ $\widetilde{X},(G, E)$ is a soft $\alpha$-open set but not a soft A-set.

Theorem 3.3. Let $(X, \tau, E)$ be a soft topological space. A soft set $(F, E)$ over $X$ is soft open if and only if it is both a soft $\alpha$-open set and $a$ soft $A$-set in $X$.

Proof. Necessity is trivial.

To prove the sufficiency, let $(F, E)=(G, E) \sqcap(K, E)$ be a soft A-set, where $(G, E)$ is soft open and $(K, E)=$ $\operatorname{cl}(\operatorname{int}(K, E))$. Since $(F, E)$ is a soft $\alpha$-open set, we have $(G, E) \sqcap(K, E) \sqsubseteq \operatorname{int}(\operatorname{cl}(\operatorname{int}((G, E) \sqcap(K, E))))=$ $\operatorname{int}(\operatorname{cl}(\operatorname{int}(G, E) \sqcap \operatorname{int}(K, E)))=\operatorname{int}(\operatorname{cl}((G, E) \sqcap \operatorname{int}(K, E))) \sqsubseteq \operatorname{int}(\operatorname{cl}(G, E) \sqcap \operatorname{cl}(\operatorname{int}(K, E)))=\operatorname{int}(\operatorname{cl}(G, E) \sqcap$ $(K, E))=\operatorname{int}(\operatorname{cl}(G, E)) \sqcap \operatorname{int}(K, E)$.

Since $(G, E) \sqsubseteq \operatorname{int}(\operatorname{cl}(G, E))$, we have $(G, E) \sqcap(K, E)=((G, E) \sqcap(K, E)) \sqcap(G, E) \sqsubseteq \operatorname{int}(\operatorname{cl}(G, E)) \sqcap \operatorname{int}(K, E) \sqcap$ $(G, E)=(G, E) \sqcap \operatorname{int}(K, E)$. Notice $(G, E) \sqcap(K, E) \sqsupseteq(G, E) \sqcap \operatorname{int}(K, E)$, we have $(G, E) \sqcap(K, E)=(G, E) \sqcap$ $\operatorname{int}(K, E)$, therefore $(F, E)=(G, E) \sqcap(K, E)$ is a soft open set.

Definition 3.2. Let $(X, \tau, E)$ be a soft topological space. A soft set $(F, E)$ is called soft $t$-set in $X$ if $\operatorname{int}(c l(F, E))=$ $\operatorname{int}(F, E)$.

We have an immediate result from Definition 3.2.

Theorem 3.4. In a soft topological space $(X, \tau, E)$, every soft closed set is a soft $t$-set.

The converse of Theorem 3.4 is not true. In fact we have the following result.

Theorem 3.5. In a soft topological space $(X, \tau, E)$, every soft regular open set is a soft $t$-set.

In a soft topological space $(X, \tau, E)$, a soft open set need not be a soft $t$-set as shown in the following example.

Example 3.5. Let $X=\left\{x_{1}, x_{2}, x_{3}\right\}, E=\left\{e_{1}, e_{2}\right\}$ and $\tau=\left\{\Phi, \tilde{X},\left(F_{1}, E\right),\left(F_{2}, E\right)\right\}$ where $\left(F_{1}, E\right),\left(F_{2}, E\right)$ are soft sets over $X$, defined as follows:

$$
\begin{aligned}
& \left(F_{1}, E\right)=\left\{\left(e_{1},\left\{x_{1}\right\}\right),\left(e_{2}, \emptyset\right)\right\}, \\
& \left(F_{2}, E\right)=\left\{\left(e_{1},\left\{x_{1}, x_{2}\right\}\right),\left(e_{2}, \emptyset\right)\right\} .
\end{aligned}
$$

Then $\tau$ defines a soft topology on $X$ and thus $(X, \tau, E)$ is a soft topological space over $X$. Then $\left(F_{1}, E\right)$ is soft open but not a soft t-set.

Theorem 3.6. Let $(X, \tau, E)$ be a soft topological space. If $(F, E)$ and $(G, E)$ are two soft $t$-sets in $X$, then $(F, E) \sqcap(G, E)$ is a soft $t$-set.

Proof. $\operatorname{int}((F, E) \sqcap(G, E)) \sqsubseteq \operatorname{int}(\operatorname{cl}((F, E) \sqcap(G, E))) \sqsubseteq \operatorname{int}(\operatorname{cl}(F, E) \sqcap \operatorname{cl}(G, E))=\operatorname{int}(\operatorname{cl}(F, E)) \sqcap \operatorname{int}(\operatorname{cl}(G, E))=$ $\operatorname{int}(F, E) \sqcap \operatorname{int}(G, E)=\operatorname{int}((F, E) \sqcap(G, E))$.

Theorem 3.7. Let $(X, \tau, E)$ be a soft topological space. A soft set $(F, E)$ is soft regular open in $X$ if and only if $(F, E)$ is both a soft preopen set and $a$ soft $t$-set.

Proof. The proof is obtained using $\operatorname{int}(F, E) \sqsubseteq(F, E) \sqsubseteq \operatorname{int}(c l(F, E))=\operatorname{int}(F, E)$.

Definition 3.3. Let $(X, \tau, E)$ be a soft topological space. A soft set $(F, E)$ is called soft B-set in $X$ if $(F, E)=$ $(G, E) \sqcap(H, E)$, where $(G, E)$ is a soft open set and $(H, E)$ is a soft $t$-set in $X$.

Theorem 3.8. In a soft topological space $(X, \tau, E)$, every soft $t$-set is a soft B-set. 
Proof. Let $(F, E)$ be a soft $t$-set in $X$. Since $(F, E) \sqcap \tilde{X}=(F, E)$, the proof is obvious.

Theorem 3.9. In a soft topological space $(X, \tau, E)$, every soft open set is a soft B-set.

Proof. Let $(F, E)$ be a soft open set in $X .(F, E)=(F, E) \sqcap \tilde{X}$ such that $\operatorname{int}(\operatorname{cl}(\tilde{X}))=\operatorname{int}(\tilde{X})=\tilde{X}$.

The converse of Theorem 3.9 is not true in general as shown in the following example.

Example 3.6. Let $X=\left\{x_{1}, x_{2}, x_{3}, x_{4}\right\}$ and $E=\left\{e_{1}, e_{2}\right\}$. Let us take the soft topology $\tau$ on $X$ in Example 3.1. Let $(G, E)$ be a soft set over $X$ such that $(G, E)=\left\{\left(e_{1},\left\{x_{3}, x_{4}\right\}\right),\left(e_{2},\left\{x_{2}\right\}\right)\right\}$. Since $\operatorname{int}(\operatorname{cl}(G, E))=\operatorname{int}(G, E)=\emptyset$, $(G, E)$ is a soft t-set. Let $(H, E)$ be a soft set over $X$ such that $(H, E)=\left\{\left(e_{1},\left\{x_{4}\right\}\right),\left(e_{2},\left\{x_{2}\right\}\right)\right\}$. Since $(H, E)=$ $\left(F_{5}, E\right) \sqcap(G, E)$ where $\left(F_{5}, E\right)$ is soft open and $(G, E)$ is a soft t-set, $(H, E)$ is a soft B-set but not soft open. Also, $(G, E)$ is a soft B-set since every soft t-set is a soft B-set but not soft open.

From Theorem 3.4 and Theorem 3.8, we have

Theorem 3.10. In a soft topological space $(X, \tau, E)$, every soft closed set is a soft B-set.

From Theorem 3.4, we have

Theorem 3.11. In a soft topological space $(X, \tau, E)$, every soft $A$-set is a soft B-set.

The converse of Theorem 3.11 is not true in general as shown in the following example.

Example 3.7. Let $X=\left\{x_{1}, x_{2}, x_{3}, x_{4}\right\}$ and $E=\left\{e_{1}, e_{2}\right\}$. Let us take the soft topology $\tau$ on $X$ in Example 3.1 and the soft set $(G, E)=\left\{\left(e_{1},\left\{x_{3}, x_{4}\right\}\right),\left(e_{2},\left\{x_{2}\right\}\right)\right\}$ in Example 3.6. Then $(G, E)$ is a soft B-set but not a soft A-set.

The examples below show that a soft B-set need not be a soft preopen set and a soft preopen set need not be a soft B-set.

Example 3.8. Let $X=\left\{x_{1}, x_{2}, x_{3}, x_{4}\right\}$ and $E=\left\{e_{1}, e_{2}\right\}$. Let us take the soft topology $\tau$ on $X$ in Example 3.1 and the soft set $(H, E)=\left\{\left(e_{1},\left\{x_{4}\right\}\right),\left(e_{2},\left\{x_{2}\right\}\right)\right\}$ in Example 3.6. Then $(H, E)$ is a soft B-set but not soft preopen.

Example 3.9. Let $X=\left\{x_{1}, x_{2}, x_{3}\right\}$ and $E=\left\{e_{1}, e_{2}\right\}$. Let us take the soft topology $\tau$ on $X$ and the soft set $(G, E)=\left\{\left(e_{1},\left\{x_{1}, x_{2}\right\}\right),\left(e_{2},\left\{x_{2}\right\}\right)\right\}$ in Example 3.4. Then $(G, E)$ is soft preopen since every soft $\alpha$-open set is a soft preopen set but not a soft B-set.

The examples below show that a soft B-set need not be a soft semiopen set and a soft semiopen set need not be a soft B-set.

Example 3.10. Let $X=\left\{x_{1}, x_{2}, x_{3}, x_{4}\right\}$ and $E=\left\{e_{1}, e_{2}\right\}$. Let us take the soft topology $\tau$ on $X$ in Example 3.1 and the soft set $(G, E)=\left\{\left(e_{1},\left\{x_{3}, x_{4}\right\}\right),\left(e_{2},\left\{x_{2}\right\}\right)\right\}$ in Example 3.6. Then $(G, E)$ is a soft B-set but not soft semiopen.

Example 3.11. Let $X=\left\{x_{1}, x_{2}, x_{3}\right\}$ and $E=\left\{e_{1}, e_{2}\right\}$. Let us take the soft topology $\tau$ on $X$ and the soft set $(G, E)=\left\{\left(e_{1},\left\{x_{1}, x_{2}\right\}\right),\left(e_{2},\left\{x_{2}\right\}\right)\right\}$ in Example 3.4. Then $(G, E)$ is soft semiopen since every soft $\alpha$-open set is a soft semiopen set but not a soft B-set.

Theorem 3.12. Let $(X, \tau, E)$ be a soft topological space. A soft set $(F, E)$ over $X$ is soft open if and only if it is both a soft preopen set and a soft $B$-set.

Proof. Necessity is trivial, we prove the sufficiency. Since $(F, E)$ is a soft B-set, we have $(F, E)=(G, E) \sqcap(H, E)$, where $(G, E)$ is a soft open set and $\operatorname{int}(\operatorname{cl}(H, E))=\operatorname{int}(H, E)$. Since $(F, E)$ is soft preopen, we have $(F, E) \sqsubseteq$ $\operatorname{int}(\operatorname{cl}(F, E))=\operatorname{int}(\operatorname{cl}((G, E) \sqcap(H, E))) \sqsubseteq \operatorname{int}(\operatorname{cl}(G, E) \sqcap \operatorname{cl}(H, E))=\operatorname{int}(\operatorname{cl}(G, E)) \sqcap \operatorname{int}(\operatorname{cl}(H, E))=\operatorname{int}(\operatorname{cl}(G, E)) \sqcap$ $\operatorname{int}(H, E)$.

Hence $(F, E)=(G, E) \sqcap(H, E)=((G, E) \sqcap(H, E)) \sqcap(G, E) \sqsubseteq(\operatorname{int}(\operatorname{cl}(G, E)) \sqcap \operatorname{int}(H, E)) \sqcap(G, E)=$ $(\operatorname{int}(c l(G, E)) \sqcap(G, E)) \sqcap \operatorname{int}(H, E)=(G, E) \sqcap \operatorname{int}(H, E)$. Notice $(F, E)=(G, E) \sqcap(H, E) \sqsupseteq(G, E) \sqcap \operatorname{int}(H, E)$, we have $(F, E)=(G, E) \sqcap \operatorname{int}(H, E)$. Thus we obtain $(F, E)$ is soft open.

We have the following implications for a soft topological space $(X, \tau, E)$. These implications are not reversible.

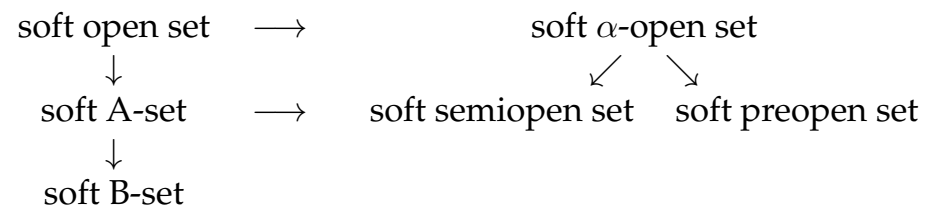




\section{Soft A-continuity and Soft B-continuity}

Definition 4.1. Let $(X, \tau, E)$ and $(Y, v, K)$ be soft topological spaces. Let $u: X \longrightarrow Y$ and $p: E \longrightarrow K$ be mappings. Let $f_{p u}: S S(X)_{E} \longrightarrow S S(Y)_{K}$ be a function. Then

(1) $f_{p u}$ is called a soft A-continuous function if for each $(G, K) \in S O S(Y), f_{p u}^{-1}(G, K)$ is a soft A-set in $X$.

(2) $f_{p u}$ is called a soft B-continuous function if for each $(G, K) \in S O S(Y), f_{p u}^{-1}(G, K)$ is a soft B-set in $X$.

Example 4.1. Let $X=\left\{x_{1}, x_{2}, x_{3}, x_{4}\right\}, Y=\left\{y_{1}, y_{2}, y_{3}\right\}, E=\left\{e_{1}, e_{2}\right\}, K=\left\{k_{1}, k_{2}\right\}, \tau=\left\{\Phi, \widetilde{X},\left(F_{1}, E\right), \ldots,\left(F_{11}, E\right)\right\}$ in Example 3.1, $v=\{\Phi, \tilde{Y},(H, K)\}$ such that $(H, K)=\left\{\left(k_{1},\left\{y_{1}, y_{2}\right\}\right),\left(k_{2},\left\{y_{1}\right\}\right)\right\}$ and $(X, \tau, E)$ and $(Y, v, K)$ be soft topological spaces.

Define $u: X \longrightarrow Y$ and $p: E \longrightarrow K$ as

$u\left(x_{1}\right)=u\left(x_{4}\right)=\left\{y_{3}\right\}, u\left(x_{2}\right)=\left\{y_{2}\right\}, u\left(x_{3}\right)=\left\{y_{1}\right\}$ and $p\left(e_{1}\right)=\left\{k_{1}\right\}, p\left(e_{2}\right)=\left\{k_{2}\right\}$.

Let $f_{p u}: S S(X)_{E} \longrightarrow S S(Y)_{K}$ be a function. Then $(H, K)$ is soft open in $Y$ and $f_{p u}^{-1}(H, K)=(G, E)=$ $\left\{\left(e_{1},\left\{x_{2}, x_{3}\right\}\right),\left(e_{2},\left\{x_{3}\right\}\right)\right\}$ is a soft A-set in $X$ by Example 3.1. Therefore, $f_{p u}$ is a soft A-continuous function.

Example 4.2. Let $X=\left\{x_{1}, x_{2}, x_{3}, x_{4}\right\}, Y=\left\{y_{1}, y_{2}, y_{3}\right\}, E=\left\{e_{1}, e_{2}\right\}, K=\left\{k_{1}, k_{2}\right\}, \tau=\left\{\Phi, \tilde{X},\left(F_{1}, E\right), \ldots,\left(F_{11}, E\right)\right\}$ in Example 3.1, $v=\{\Phi, \tilde{Y},(G, K)\}$ such that $(G, K)=\left\{\left(k_{1},\left\{y_{1}\right\}\right),\left(k_{2},\left\{y_{3}\right\}\right)\right\}$ and $(X, \tau, E)$ and $(Y, v, K)$ be soft topological spaces.

Define $u: X \longrightarrow Y$ and $p: E \longrightarrow K$ as

$u\left(x_{1}\right)=u\left(x_{3}\right)=\left\{y_{2}\right\}, u\left(x_{2}\right)=\left\{y_{1}\right\}, u\left(x_{4}\right)=\left\{y_{3}\right\}$ and $p\left(e_{1}\right)=\left\{k_{2}\right\}, p\left(e_{2}\right)=\left\{k_{1}\right\}$.

Let $f_{p u}: S S(X)_{E} \longrightarrow S S(Y)_{K}$ be a function. Then $(G, K)$ is soft open in $Y$ and $f_{p u}^{-1}(G, K)=(H, E)=$ $\left\{\left(e_{1},\left\{x_{4}\right\}\right),\left(e_{2},\left\{x_{2}\right\}\right)\right\}$ is a soft B-set in $X$ by Example 3.6. Therefore, $f_{p u}$ is a soft B-continuous function.

By Theorem 3.1 and Theorem 3.2, we have an immediate result.

Remark 4.1. Every soft continuous function is soft A-continuous and every soft A-continuous function is soft semicontinuous.

By Theorem 3.9, we have an immediate result.

Remark 4.2. Every soft continuous function is soft B-continuous.

By Theorem 3.11, we have an immediate result.

Theorem 4.1. Let $(X, \tau, E)$ and $(Y, v, K)$ be soft topological spaces and $f_{p u}: S S(X)_{E} \longrightarrow S S(Y)_{K}$ be a function. Then, every soft A-continuous function is soft B-continuous.

By Theorem 3.3, we have the following decomposition of soft continuity:

Theorem 4.2. Let $(X, \tau, E)$ and $(Y, v, K)$ be soft topological spaces and $f_{p u}: S S(X)_{E} \longrightarrow S S(Y)_{K}$ be a function. Then $f_{p u}$ is a soft continuous function if and only if it is both soft $\alpha$-continuous and soft A-continuous.

Corollary 4.1. Let $(X, \tau, E)$ and $(Y, v, K)$ be soft topological spaces and $f_{p u}: S S(X)_{E} \longrightarrow S S(Y)_{K}$ be a function. Then $f_{p u}$ is soft continuous if and only if it is both soft precontinuous and soft A-continuous.

Corollary 4.2. Let $(X, \tau, E)$ and $(Y, v, K)$ be soft topological spaces and $f_{p u}: S S(X)_{E} \longrightarrow S S(Y)_{K}$ be a function. If $f_{p u}$ is both soft precontinuous and soft $A$-continuous, then it is soft $\alpha$-continuous.

It is clear that Corollary 4.1 is a generalization of Theorem 4.2 . We observe that Corollary 4.2 follows from the fact that soft A-continuous functions are soft semicontinuous from Remark 4.1 and a function is soft $\alpha$-continuous if it is soft precontinuous and soft semicontinuous from Theorem 2.3.

By Theorem 3.12, we have the following decomposition of soft continuity:

Theorem 4.3. Let $(X, \tau, E)$ and $(Y, v, K)$ be soft topological spaces and $f_{p u}: S S(X)_{E} \longrightarrow S S(Y)_{K}$ be a function.Then $f_{p u}$ is a soft continuous function if and only if it is both soft precontinuous and soft B-continuous.

We have the following implications.

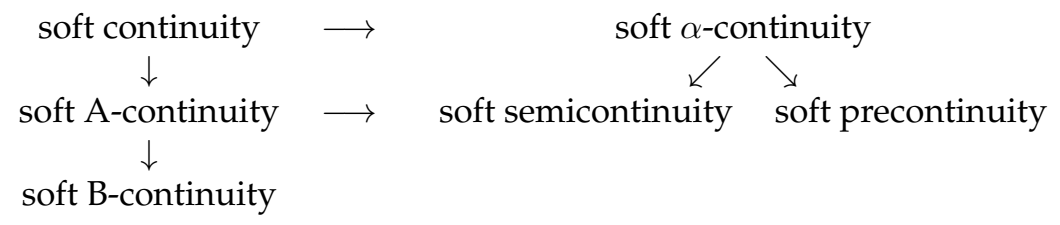




\section{References}

[1] Akdağ, M. and Özkan, A., Soft $\alpha$-open sets and soft $\alpha$-continuous functions. Abstract and Applied Analysis http://dx.doi.org/10.1155/2014/891341, (2014).

[2] Arockiarani, I. and Arokialancy, A. Generalized soft $\mathrm{g} \beta$-closed sets and soft gs $\beta$-closed sets in soft topological spaces. International Journal of Mathematical Archive 4 (2013), no. 2, 1-7.

[3] Aygünoğlu, A. and Aygün, H., Some notes on soft topological spaces. Neural Computing and Applications 21 (2012), no. 1, 113-119.

[4] Chen, B., Soft semi-open sets and related properties in soft topological spaces. Applied Mathematics $\mathcal{E}$ Information Sciences 7 (2013), no. 1, 287-294.

[5] Hussain, S. and Ahmad, B., Some properties of soft topological spaces. Computers and Mathematics with Applications 62 (2011), no. 11, 4058-4067.

[6] Kharal, A. and Ahmad, B., Mappings on soft classes. New Mathematics and Natural Computation 7 (2011), no. 3, 471-481.

[7] Mahanta, J. and Das, P.K., On soft topological space via semi-open and semi-closed soft sets. Cornell University Library arXiv:1203.4133, (2012).

[8] Maji, P.K., Biswas, R. and Roy, A.R., Soft set theory. Computers and Mathematics with Applications 45 (2003), no. $4-5,555-562$.

[9] Min, W.K., A note on soft topological spaces. Computers and Mathematics with Applications 62 (2011), no. 9, 3524-3528.

[10] Molodtsov, D., Soft set theory-first results. Computers and Mathematics with Applications 37 (1999), no. 4-5, 19-31.

[11] Shabir, M. and Naz, M., On soft topological spaces. Computers and Mathematics with Applications 61 (2011), no. 7, 1786-1799.

[12] Yüksel, Ş., Tozlu, N. and Güzel Ergül, Z., Soft regular generalized closed sets in soft topological spaces. International Journal of Mathematical Analysis 8 (2014), no. 8, 355-367.

[13] Zorlutuna, I., Akdağ, M., Min, W.K. and Atmaca, S., Remarks on soft topological spaces. Annals of Fuzzy Mathematics and Informatics 3 (2012), no. 2, 171-185.

\section{Affiliations}

NAİME TOZLU

AdDRESS: Ömer Halisdemir University, Dept. of Mathematics, 51240, Niğde-Turkey.

E-MAIL: naimetozlu@ohu.edu.tr

ŞAZIYYE YÜKSEL

AdDress: Selçuk University, Dept. of Mathematics, 42100, Konya-Turkey.

E-MAIL: sayuksel@yahoo.com 